\title{
Joint Coordinated Beamforming and Admission Control for Fronthaul Constrained Cloud-RANs
}

\author{
Vu Nguyen Ha and Long Bao Le
}

\begin{abstract}
In this paper, we consider the joint coordinated beamforming and admission control design for cloud radio access networks (Cloud-RANs). Specifically, the set of multi-antenna remote radio heads (RRHs) serving each single-antenna user and the corresponding beamforming vectors are optimized to minimize the total transmission power subject to constraints on the capacity of fronthaul links, maximum powers of RRHs, and the minimum signal to interference plus noise ratios (SINRs) of users. Since the minimum SINR requirements of all users may not be guaranteed, some users may need to be removed so that all constraints can be satisfied. This NP-hard beamforming and admission control problem can be typically solved via a greedy algorithm. We instead propose a novel convex relaxation approach to formulate the underlying problem to a single-stage semi-definite program (SDP) based on which we develop an iterative algorithm to solve it. We then present numerical results to demonstrate the significant gains of the proposed algorithm compared to the greedy counterpart. Also, the impacts of the target SINR and cluster size on the number of supported users and total transmission power are also studied.
\end{abstract}

Index Terms-Cloud radio access network (Cloud-RAN), beamforming, power allocation, admission control, resource allocation, clustering.

\section{INTRODUCTION}

Coordinated multipoint (CoMP) transmission and reception techniques have been considered as important solutions to enhance the capacity and coverage performance of wireless cellular networks [1]. Deployment of CoMP schemes typically requires high-capacity backhaul links connecting different base stations (BSs) for various CSI and information exchanges [2]. In addition, heavy computation and processing are usually demanded for the BSs and user equipment (UE) by CoMP schemes. Cloud-RAN has recently been proposed as an alternative architecture where most processing functions are conducted in the cloud, which allows more efficient utilization of computational and radio resources [3]. Cloud-RAN can also help deploy other emerging technologies such as small-cells efficiently $[4,5]$.

In the typical deployment of Cloud-RAN, the digital baseband processing units (BBUs) are implemented in the cloud to perform various signal processing functions while RRHs transmit RF signals to the users using baseband signals from the cloud. BBUs are connected with RRHs via high-speed fronthaul links that enable information exchanges between the cloud and RRHs. Since these fronthaul links have finite capacity, the design of communications and signal processing schemes must account for the fronthaul capacity constraints. In [6], compression techniques were considered to reduce the amount of data transmitted over the backhaul links. In [7],

The authors are with INRS, University of Quebec, Montreal, QC, Canada; e-mails: \{hanguyen,long.le\}@emt.inrs.ca. the authors derived the achievable rate region for two-BS case where the backhaul links utilized for information sharing with the BSs have limited capacity. The design of beamforming in Cloud-RAN to minimize the energy consumption of the RRHs and fronthaul network was considered in [8]. This work, however, did not consider the limited capacity constraints of fronthaul links. Finally, the works $[9,10]$ proposed greedy algorithms to deal with limited fronthaul constraints in coordinated communications design for Cloud-RAN.

In this paper, we consider the joint downlink coordinated beamforming and admission control design that aims to minimize the total transmission power subject to practical constraints on the RRH maximum power, users' QoS, and fronthaul capacity. We propose to formulate this joint problem to a single-stage optimization problem which is further transformed into a convex problem via a novel relaxation approach. Then, we develop an iterative algorithm to solve it. We also describe a fast greedy algorithm where users are gradually removed until all constraints can be satisfied. Numerical results confirm that our proposed algorithm achieves significantly better performance in terms of number of supported users and transmission power that the greedy algorithm. In addition, we show the impacts of the target SINR, fronthaul capacity, and maximum cluster size on the network performance for both algorithms.

For notations, we use $\mathbf{X}^{T}, \mathbf{X}^{H}, \operatorname{Tr}(\mathbf{X})$ and $\operatorname{rank}(\mathbf{X})$ to denote transpose, Hermitian transpose, trace, and rank of matrix X, respectively. $\mathbf{1}_{x \times y}, \mathbf{0}_{x \times y}$ denote the matrix of ones, matrix of zeros whose dimension are $x \times y$, respectively. $|\mathcal{S}|$ denotes the cardinality of set $\mathcal{S}$ and $\operatorname{diag}(\mathbf{x})$ is the diagonal matrix constructed from the elements of vector $\mathbf{x}$.

\section{System Model AND Problem Formulation}

\section{A. System Model}

We consider downlink communications in the Cloud-RAN with $K \mathrm{RRH}$, which coordinate to transmit to $M$ UEs. In addition, base-band signals are processed in the cloud BBU pool. Moreover, the BBUs pool is assumed to be connected with RRHs via finite capacity fronthaul links. This CloudRAN architecture is illustrated in Fig. 1,

Let $\mathcal{K}$ and $\mathcal{U}$ be the sets of RRHs and UEs in the network, respectively. We consider the MISO transmission from RRHs to UEs where RRH $k$ is equipped with $N_{k}$ antennas and each UE has a single antenna. We assume that each UE is served by a specific group of RRHs, which receive corresponding baseband signals from the cloud. This coordinated transmission scheme aims to exploit antenna resources available at different RRHs to mitigate the interference [1]. Let us now define binary variables $t_{u}^{k}$ where $t_{u}^{k}=1$ if RRH $k$ serves UE 


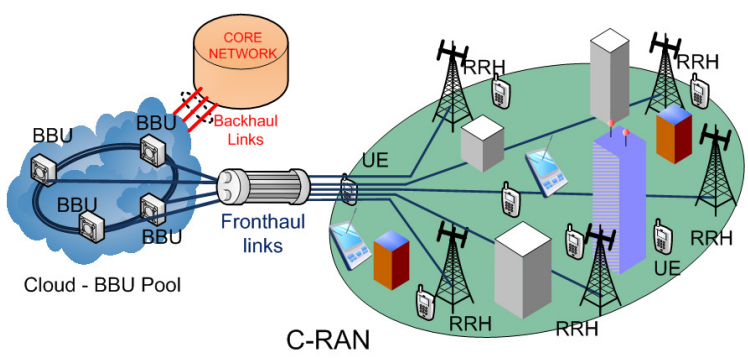

Fig. 1. The Cloud-RAN architecture.

$u$ and $t_{u}^{k}=0$ otherwise. Then, the set of RRHs that serve UE $u$ can be determined as

$$
\mathcal{R}_{u}=\left\{k \mid k \in \mathcal{R}, t_{u}^{k}=1\right\} .
$$

The RRH allocation solution, is, therefore, represented by $\left\{\mathcal{R}_{1}, \mathcal{R}_{2}, \ldots, \mathcal{R}_{M}\right\}$. Also, the cloud receives data of each UE $u$ from the core network, produces baseband signals, and determine the precoding vectors for the corresponding RRHs. Then, it sends the baseband signals and precoding vectors of each UE $u$ to the serving RRHs in the set $\mathcal{R}_{u}$, which upconvert the received baseband signals to the RF band and transmit to corresponding UEs using the chosen precoding vectors.

We assume that UE $u$ receives symbol sequence $x_{u} \in \mathbb{C}$ of unit power, which is transmitted by RRHs in set $\mathcal{R}_{u}$ upon receiving the processed baseband signals from the cloud. Let $\mathbf{v}_{u}^{k} \in \mathbb{C}^{N_{l} \times 1}$ be the precoding vector at RRH $k$ for the signal transmitted to UE $u$. Then, the corresponding transmission power can be expressed as

$$
p_{u}^{k}=\mathbf{v}_{u}^{k H} \mathbf{v}_{u}^{k}
$$

Let $\mathbf{p}^{k}=\left[p_{1}^{k}, \ldots, p_{M}^{k}\right]$ be the transmission power vector of RRH $k$. Here, if $p_{u}^{k}=0$ then RRH $k$ does not serve UE $u$, which implies that the fronthaul link from the cloud to RRH $k$ does not consume any capacity for carrying the baseband signal of UE $u$. Hence, the transmission power vector $\mathbf{p}^{k}$ also indicates the number of baseband signals carried by the fronthaul links, which corresponds to the number of its non-zeros elements. Mathematically, the number of baseband signals carried by the fronthaul link connecting the cloud and the RRH $k$ can be written as

$$
C_{k}\left(\mathbf{p}^{k}\right)=\left\|\mathbf{p}^{k}\right\|_{\mathbf{0}}
$$

where $\|\mathbf{x}\|_{\mathbf{0}}$ indicates the $\ell 0$-norm of vector $\mathbf{x}$. Given the RRH allocation solution, the baseband signal $y_{u}$ received at UE $u$ can be written as

$$
y_{u}=\underbrace{\sum_{k \in \mathcal{R}_{u}} \mathbf{h}_{u}^{k H} \mathbf{v}_{u}^{k} x_{u}}_{\text {desired signal }}+\underbrace{\sum_{i=1, \neq u}^{M} \sum_{l \in \mathcal{R}_{i}} \mathbf{h}_{u}^{l H} \mathbf{v}_{i}^{l} x_{i}}_{\text {interference }}+z_{u}
$$

where $\mathbf{h}_{u}^{k} \in \mathbb{C}^{N_{k} \times 1}$ denotes the channel coefficients between RRH $k$ and UE $u$, and $z_{u}$ describes the noise at UE $u$. Also, the SINR achieved by UE $u$ is

$$
\Gamma_{u}(\mathbf{V})=\frac{\left|\sum_{k \in \mathcal{R}_{u}} \mathbf{h}_{u}^{k H} \mathbf{v}_{u}^{k}\right|^{2}}{\sum_{i=1, \neq u}^{M}\left|\sum_{l \in \mathcal{R}_{i}} \mathbf{h}_{u}^{l H} \mathbf{v}_{i}^{l}\right|^{2}+\sigma^{2}}
$$

where $\sigma^{2}$ is the noise power and $\mathbf{V}$ is the precoding matrix whose columns are precoding vectors $\mathbf{v}_{u}^{k}, k \in \mathcal{R}_{u}$.

\section{B. RRH Clustering Constraints}

To limit the computational complexity in large networks, we can limit that each UE may only be served by certain nearby RRHs. Denote $\mathcal{K}_{u}\left(\mathcal{K}_{u} \subseteq \mathcal{K}\right)$ as the maximum set of RRHs which are allowed to serve UE $u$. Accordingly, we can also define the set of UEs which can be served by RRH $k$ as

$$
\mathcal{U}_{k}=\left\{u \mid u \in \mathcal{U}, k \in \mathcal{K}_{u}\right\}
$$

Under these clustering constraints, RRH $k$ can only receive the baseband signals of UEs in set $\mathcal{U}_{k}$ from the cloud. Hence, RRH $k$ can serve at most $C_{k}^{\max }=\left|\mathcal{U}_{k}\right|$ UEs.

\section{Joint Coordinated Beamforming and Admission Control Problem}

Our design aims to determine the set of RRHs serving each $\mathrm{UE}$ and the corresponding precoding vectors for all RRHs (i.e., represented by $\mathbf{V}$ ) to minimize the total transmission power under the constrained fronthaul capacity, limited transmission power, and users' QoS. Here, the required QoS of UE $u$ is described as

$$
\Gamma_{u}(\mathbf{V}) \geq \bar{\gamma}_{u}, \quad \forall u \in \mathcal{U}
$$

where $\bar{\gamma}_{u}$ denotes the target SINR. We also impose the constraint on the total transmission power of RRH $k$ by its maximum power budget $P_{k}(k \in \mathcal{K})$, which is described as

$$
\sum_{u \in \mathcal{U}} p_{u}^{k}=\left\|\mathbf{p}^{k}\right\|_{\mathbf{1}}=\sum_{u \in \mathcal{U}} \mathbf{v}_{u}^{k H} \mathbf{v}_{u}^{k} \leq P_{k}, \quad \forall k \in \mathcal{K}
$$

where $\|\mathbf{x}\|_{1}$ indicates the $\ell 1$-norm of vector $\mathbf{x}$. We assume that the fronthaul link between the cloud and RRH $k$ is capable of carrying at most $\bar{C}_{k}$ baseband signals for UEs. This is transferred into the following fronthaul capacity constraints

$$
C_{k}\left(\mathbf{p}^{k}\right)=\left\|\mathbf{p}^{k}\right\|_{\mathbf{0}} \leq \bar{C}_{k}, \quad k \in \mathcal{K}
$$

where this constraint is only imposed for RRH $k$ if $\bar{C}_{k} \leq C_{k}^{\max }$ (i.e., if $\bar{C}_{k}>C_{k}^{\max }$, there is no fronthaul capacity constraint for RRH $k$ ).

Since it may not be possible to satisfy the constraints (7), (8), (9) for all users, we may need to select a smallest set of UEs to remove so as to maintain these constraints (i.e., perform user admission control). Let us now define a binary admission control variable $s_{u}$ for user $u(\forall u \in \mathcal{U})$ where $s_{u}=-1$ if user $u$ is admitted (supported) and $s_{u}=1$, otherwise. Then, the set of non-supported users can be defined as $\mathcal{S}=\left\{u \mid u \in \mathcal{U}, s_{u}=1\right\}$. Therefore, our design problem 
would be addressed in two stages where the admission control is performed in the first stage to solve the following problem

$$
\begin{aligned}
\min _{\mathbf{V},\left\{\mathbf{p}^{k}\right\},\left\{s_{u}\right\}} & |\mathcal{S}| \\
\text { s.t. } & \text { constraints (2), (8), (9), } \\
& \Gamma_{u}(\mathbf{V}) \geq \bar{\gamma}_{u}, \quad \forall u \in \mathcal{U} / \mathcal{S} .
\end{aligned}
$$

Given the optimal removal set $\mathcal{S}^{*}$ obtained in the first stage, we solve the following coordinated beamforming problem in the second stage

$$
\begin{aligned}
\min _{\mathbf{V},\left\{\mathbf{p}^{k}\right\}} & \sum_{k \in \mathcal{K}}\left\|\mathbf{p}^{k}\right\|_{\mathbf{1}} \\
\text { s.t. } \quad \text { constraints (2), (8), (9), } & \Gamma_{u}(\mathbf{V}) \geq \bar{\gamma}_{u}, \quad \forall u \in \mathcal{U} / \mathcal{S}^{*} .
\end{aligned}
$$

Note that constraints (9) involve $\ell 0$-norm regularization, whose constraint functions are non-convex and discontinuous. In addition, the search of the removal set $\mathcal{S}$ involves the binary variables $s_{u}$. Therefore, both problems (10) and (11) are NPhard and very difficult to solve. Being inspired by the work [11], we propose to address the admission control task through solving the following single-stage problem

$$
\begin{aligned}
\min _{\mathbf{V},\left\{\mathbf{p}^{k}\right\},\left\{s_{u}\right\}} & \sum_{k \in \mathcal{K}}\left\|\mathbf{p}^{k}\right\|_{\mathbf{1}}+A|\mathcal{S}| \\
\text { s.t. } \quad \text { constraints (2), (8), (9), } & \\
& \Gamma_{u}(\mathbf{V}) \geq \bar{\gamma}_{u}, \quad \forall u \in \mathcal{U} / \mathcal{S}, \\
& s_{u}=1 \text { or }-1, \quad \forall u \in \mathcal{U}
\end{aligned}
$$

where $A$ is a design parameter, which will be discussed in the following.

\section{Properties of the Single-stage Problem}

The parameter $A$ in (12) has strong impacts on the overall system performance. We reveal some important properties of this problem in the following propositions.

Proposition 1. Let $\left(\mathbf{V}^{*},\left\{\mathbf{p}^{k *}\right\},\left\{s_{u}^{*}\right\}\right)$ be the optimal solution of problem (12) then we have

1) The solution $\left(\mathbf{V}^{*},\left\{\mathbf{p}^{k *}\right\},\left\{s_{u}^{*}\right\}\right)$ is also feasible for the two-stage problems (10) and (11).

2) If $A>\sum_{k \in \mathcal{K}} P_{k}$ then there is no $\left(\mathbf{V}^{\prime},\left\{\mathbf{p}^{k \prime}\right\},\left\{s_{u}^{\prime}\right\}\right)$ that satisfies all constraints in problem (12) and $\left|\mathcal{S}^{\prime}\right|<$ $\left|\mathcal{S}^{*}\right|$ where $\mathcal{S}^{*}=\left\{u \mid u \in \mathcal{U}, s_{u}^{*}=1\right\}$ and $\mathcal{S}^{\prime}=$ $\left\{u \mid u \in \mathcal{U}, s_{u}^{\prime}=1\right\}$.

Proof. The proof is given in Appendix A.

The results in this proposition suggest that if we set $A>\sum_{k \in \mathcal{K}} P_{k}$ then solving the single-stage problem (12) is sufficient to obtain the optimal solution for our joint coordinated beamforming and admission control problem.

Proposition 2. Consider the following problem

$$
\min _{\mathbf{V},\left\{\mathbf{p}^{k}\right\},\left\{s_{u}\right\}} \sum_{k \in \mathcal{K}}\left\|\mathbf{p}^{k}\right\|_{\mathbf{1}}+(A / 4) \sum_{u \in \mathcal{U}}\left(1+s_{u}\right)^{2}
$$

$$
\begin{aligned}
& \text { s.t. } \text { constraints }(2),(8),(9) \text {, } \\
& s_{u}=1 \text { or }-1, \forall u \in \mathcal{U} \text {, } \\
& \frac{\left|\sum_{k \in \mathcal{R}_{u}} \mathbf{h}_{u}^{k H} \mathbf{v}_{u}^{k}\right|^{2}+\mu\left(1+s_{u}\right)^{2}}{\sum_{i=1, \neq u}^{M}\left|\sum_{l \in \mathcal{R}_{i}} \mathbf{h}_{u}^{l H} \mathbf{v}_{i}^{l}\right|^{2}+\sigma^{2}} \geq \bar{\gamma}_{u}, \quad \forall u \in \mathcal{U} .
\end{aligned}
$$

If we have

$$
\mu>\max _{u \in \mathcal{U}} \max _{k \in \mathcal{K}}\left[\bar{\gamma}_{u}\left(\sum_{l \in \mathcal{K}} P_{l} \max _{(l \in \mathcal{K}, i \in \mathcal{U})}\left\|\mathbf{h}_{i}^{l}\right\|_{2}+\sigma^{2}\right)\right] / 4,
$$

then the above problem is equivalent to the single-stage problem (12).

Proof. The proposition can be proved by showing that in both cases with $s_{u}=1$ and $s_{u}=-1$, the two problems are equivalent. Details are omitted due to the space constraint.

The results in Proposition 2 imply that instead of solving problem (12), we can equivalently solve problem (13)-(15), which is addressed in the following.

\section{Algorithm Design}

\section{A. Convex Relaxation Based Algorithm}

The main difficulty in solving problem (13)-(15) is due to the constraints (9), which involve $\ell 0$-norm non-convex and discontinuous functions. It needs to be noted that $\ell 0$-norm of a non-negative vector can be represented by sum of all the step functions of its elements. Hence, to address this challenge, we propose to approximate the $\ell 0$-norm in (9) by utilizing continuous concave function instead of the step function. In particular, let us define $\left\|\mathbf{p}^{k}\right\|_{\mathbf{0}} \simeq \sum_{u \in \mathcal{U}} \lambda\left[\left(\alpha+p_{u}^{k}\right)^{p}-\alpha^{p}\right]$ where $0<\alpha \ll 1$ and $\lambda=\left(1-\alpha^{p}\right)^{-1}$. Then, problem (13)(15) can be approximated by the following problem

$$
\begin{aligned}
\min _{\mathbf{V},\left\{\mathbf{p}^{k}\right\},\left\{s_{u}\right\}} & \sum_{k \in \mathcal{K}}\left\|\mathbf{p}^{k}\right\|_{\mathbf{1}}+(A / 4) \sum_{u \in \mathcal{U}}\left(1+s_{u}\right)^{2} \\
\text { s.t. } \quad & \text { constraints (2), (8), (14), (15), } \\
& \sum_{u \in \mathcal{U}} R_{u}\left(p_{u}^{k}\right) \leq \bar{C}_{k}, \quad \forall k \in \mathcal{K}
\end{aligned}
$$

where $R_{u}\left(p_{u}^{k}\right)=\lambda\left[\left(\alpha+p_{u}^{k}\right)^{p}-\alpha^{p}\right]$. Here, the function $R_{u}\left(p_{u}^{k}\right)$ is concave with respect to $p_{u}^{k}$. For further simplification, we define $m_{u}^{k}=\alpha+p_{u}^{k}$. Then

$$
R_{p}^{u}\left(p_{u}^{k}\right)=\bar{R}_{p}^{u}\left(m_{u}^{k}\right)=\lambda\left[\left(m_{u}^{k}\right)^{p}-\alpha^{p}\right] .
$$

The function $\bar{R}_{p}^{u}\left(m_{u}^{k}\right)$ is concave with respect to $m_{u}^{k}$. It can be verified that the function $R_{p}^{u}\left(p_{u}^{k}\right)$ can be rewritten by using its concave duality as follows:

$$
\bar{R}_{p}^{u}\left(m_{u}^{k}\right)=\inf _{z_{u}^{k}}\left[z_{u}^{k} m_{u}^{k}-R_{p}^{* u}\left(z_{u}^{k}\right)\right]
$$

where $R_{p}^{* u}\left(z_{u}^{k}\right)$ is the concave dual of $\bar{R}_{p}^{u}\left(m_{u}^{k}\right)$ given as

$$
\begin{aligned}
R_{p}^{* u}\left(z_{u}^{k}\right) & =\inf _{w}\left[z_{u}^{k} w-\bar{R}_{p}^{u}(w)\right] \\
& =(p-1) \lambda^{1 /(1-p)}\left(z_{u}^{k} / p\right)^{p /(p-1)}+\lambda \alpha^{p} .
\end{aligned}
$$




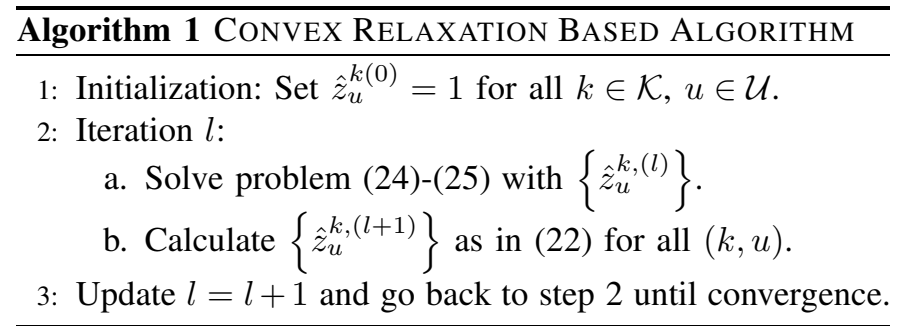

Then, by substituting (21) in (20), it is easy to find that the optimization problem in the right hand side of (20) achieves its minimum at

$$
\hat{z}_{u}^{k}=\lambda p\left(m_{u}^{k}\right)^{(p-1)}=\lambda p\left(p_{u}^{k}+\alpha\right)^{(p-1)} .
$$

With the representation of $R_{p}^{u}\left(p_{u}^{k}\right)$ in (20), the constraints (9) can be rewritten in a linear form for a given $\left\{\hat{z}_{u}^{k}\right\}$ as

$$
\sum_{u \in \mathcal{U}} \hat{z}_{u}^{k} p_{u}^{k} \leq \bar{C}_{k}+\sum_{u \in \mathcal{U}}\left[R_{p}^{* u}\left(\hat{z}_{u}^{k}\right)-\alpha \hat{z}_{u}^{k}\right], \forall k \in \mathcal{K} .
$$

In summary, for a given value of $\left\{\hat{z}_{u}^{k}\right\}$, the problem (17)-(18) can be reformulated to

$$
\min _{\mathbf{V},\left\{\mathbf{p}^{k}\right\},\left\{s_{u}\right\}} \sum_{k \in \mathcal{K}} \sum_{u \in \mathcal{U}} \mathbf{v}_{u}^{k H} \mathbf{v}_{u}^{k}+(A / 4) \sum_{u \in \mathcal{U}}\left(1+s_{u}\right)^{2}
$$

s.t. constraints (2), (8), (14), (15),

$$
\sum_{u \in \mathcal{U}} \hat{z}_{u}^{k} \mathbf{v}_{u}^{k H} \mathbf{v}_{u}^{k} \leq \bar{C}_{k}+\sum_{u \in \mathcal{U}}\left[R_{p}^{* u}\left(\hat{z}_{u}^{k}\right)-\alpha \hat{z}_{u}^{k}\right], \forall k \in \mathcal{K} .
$$

Note that problem (24)-(25) is the well-known sum-power minimization problem, which can be solved by transforming it into the SDP, which is described in Appendix C. Therefore, we can achieve our design objectives by updating $\left\{\hat{z}_{u}^{k}\right\}$ iteratively based on which we repeatedly solve the reformulated beamforming and admission control problem (24)-(25). This procedure is summarized in Algorithm 1 whose properties are stated in Proposition 3 in the following.

\section{Proposition 3. Algorithm 1 has the following properties}

1) Algorithm 1 converges.

2) The solution achieved by Algorithm 1 at convergence satisfies all constraints of problem (17)-(18).

Proof. The proof is given in Appendix B.

\section{B. Greedy Algorithm}

For comparison purposes, we describe another fast greedy algorithm in the following, which shares some spirits with algorithms proposed in [9]. We start with an empty set $\mathcal{S}$ where all UEs are admitted. Then, we iteratively solve problem (11) with the updated set $\mathcal{S}$ where each UE is served by the maximum set of RRHs with size $\left|\mathcal{K}_{u}\right|$ accounting for the clustering constraints. Then, we have to drop $C_{k}^{\max }-\bar{C}_{k}$ "weak" links between RRH $k$ and UEs in $\mathcal{U}_{k}$ to satisfy the fronthaul capacity constraint for RRH $k$. Toward this end, we propose the following metric that quantifies the relative contribution of a link between RRH $k$ and UE $u$ to the SINR of UE $u$

$$
\delta_{u}^{k}=\frac{\left|\mathbf{h}_{u}^{k H} \mathbf{v}_{u}^{k *}\right|^{2}}{\left|\sum_{l \in \mathcal{R}} \mathbf{h}_{u}^{l H} \mathbf{v}_{u}^{l *}\right|^{2}} .
$$

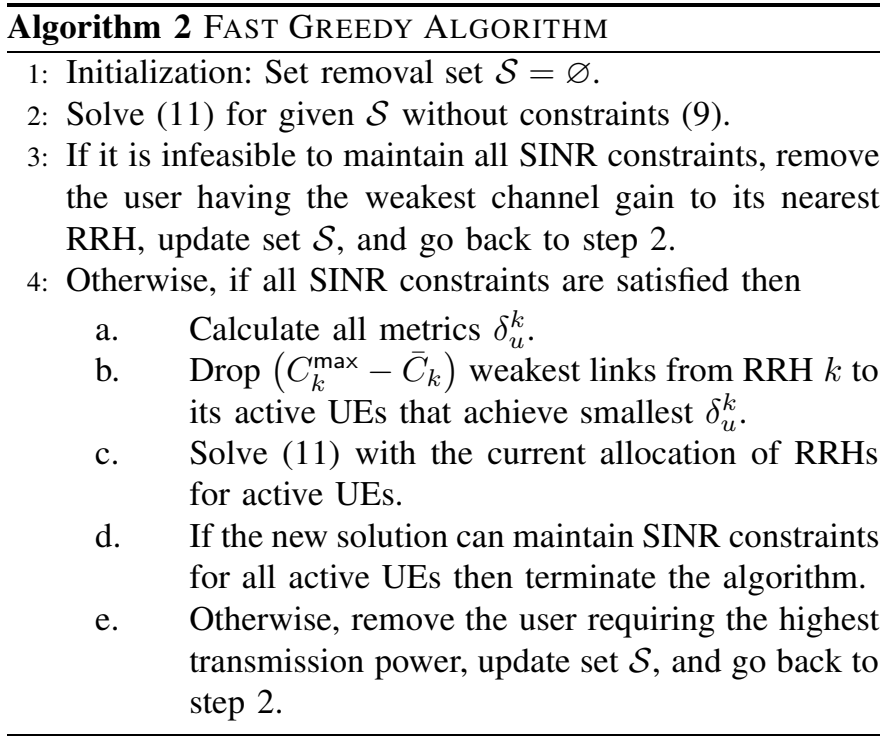

The links corresponding to low values of $\delta_{u}^{k}$ are considered "weak" ones. After dropping $C_{k}^{\max }-\bar{C}_{k}$ "weak" links for each RRH $k$, we solve problem (11) again. Then, we remove one weak UE requiring highest transmission power if it is not feasible to support all SINR constraints. These tasks are repeated until convergence. The greedy algorithm is described in Algorithm 2.

\section{Numerical Results}

We conduct numerical studies based on the 19-cell $(K=19)$ wrap-around network setup with 3 users per cell $(M=57)$ and 4 transmit antennas $\left(N_{k}=4\right)$ per RRH as illustrated in Fig. 2. UEs are randomly located inside each cell so that the distance between them and their nearest RRH is either $d$ or $d / 2$. The distance between two nearest RRHs is $2 d$ where $d=$ $250 \mathrm{~m}$. The channel gains are generated by considering both Rayleigh fading with mean value of 1 and the path loss, which is modeled as $L_{u}^{k}=36.8 \log _{10}\left(d_{u}^{k}\right)+43.8+20 \log _{10}\left(\frac{f_{c}}{5}\right)$ where $d_{u}^{k}$ is the distance from UE $u$ to RRH $k ; f_{c}=2.5 \mathrm{GHz}$. Other parameters are set as follows: noise power $\sigma^{2}=10^{-13} \mathrm{~W}$, $p=0.1$, and $P_{k}=10 \mathrm{~W}$ for all $k \in \mathcal{K}$.

In Fig. 3, we present the variations of total transmission power of all RRHs and the number of supported users versus the target SINR where we set $\bar{C}_{k}=C_{\max }=6, \forall k \in \mathcal{K}$ and $\mathcal{R}_{u}=\mathcal{K}$ for all $u \in \mathcal{U}$. As can be observed, the transmission power increases if the target SINR becomes larger and the number of supported users is unchanged. Moreover, the number of supported users decreases as the target SINR increases. It is also evident that our proposed algorithm results in larger number of supported users than that due to the greedy algorithm at the same target SINR. Moreover, if the numbers of supported users under two algorithms are similar, the greedy algorithm requires higher total transmission power.

Fig. 4 shows the number of supported users versus the target SINR for different values of fronthaul capacity per RRH where we set $\mathcal{R}_{u}=\mathcal{K}$ for all $u \in \mathcal{U}$. As expected, the number of supported users decreases as the target SINR increases and this number becomes higher with larger fronthaul capacity. Again, our proposed algorithm results in larger number of 


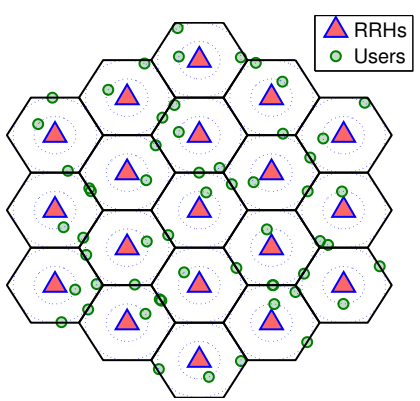

Fig. 2. Simulation network setting
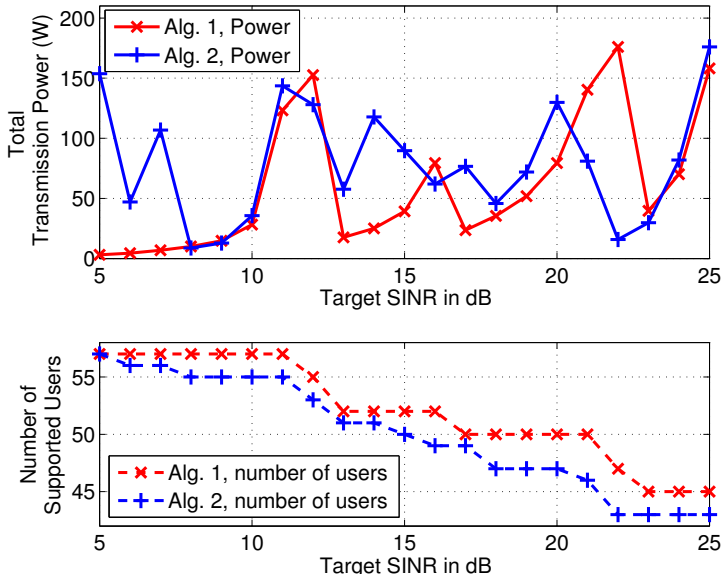

Fig. 3. Total transmission power and number of supported users versus the target SINR.

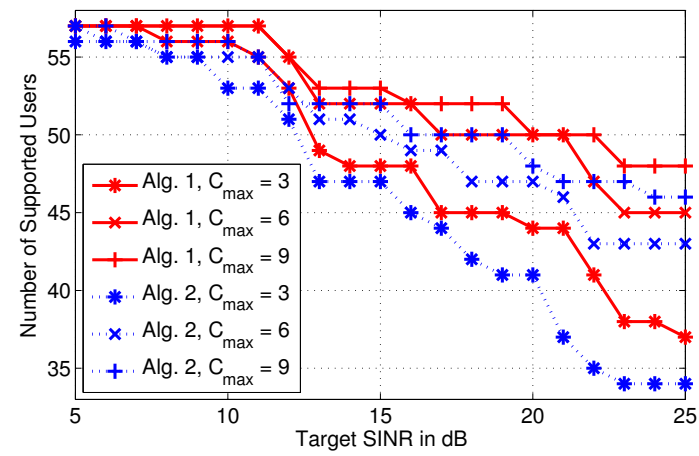

Fig. 4. Number of supported users versus the target SINR.

supported users for same target SINR and fronthaul capacity compared to the greedy counterpart. Fig. 5 illustrates how the total transmission power varies with the fronthaul capacity per RRH. As can be seen, the transmission power becomes smaller if the fronthaul capacity is larger and the number of supported users remains unchanged. For sufficiently large fronthaul capacity $\left(C_{\max }>15\right)$, the proposed algorithm results in smaller power consumption than that due to the greedy algorithm for the same or even slightly larger number of supported users. This again confirms the superiority of our proposed algorithm.

Finally, Fig. 6 represents the variations in the number of

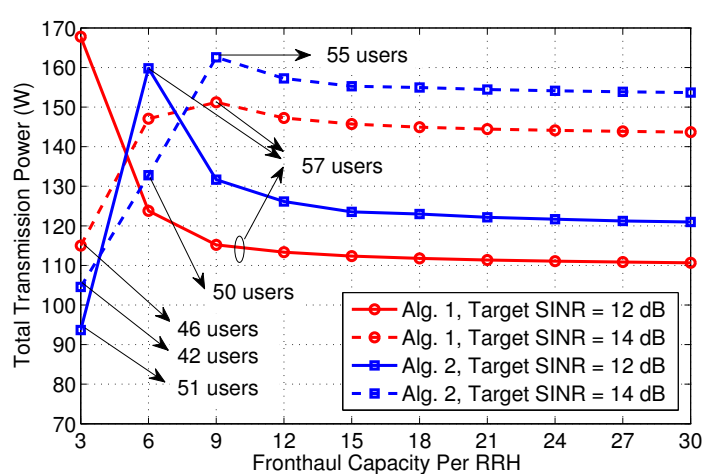

Fig. 5. Total transmission power versus the fronthaul capacity.

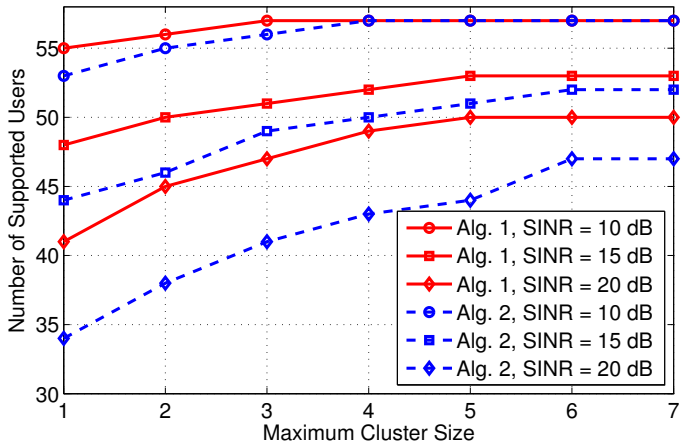

Fig. 6. Total transmission power versus maximum cluster size (maximum number of RRHs serving one user).

supported users with the cluster size (i.e., maximum number of RRHs serving one user). The figure confirms that the larger cluster size results in larger number of supported users and the gap between the proposed and greedy algorithms becomes smaller for larger cluster sizes. However, the gap tends to be larger with higher target SINR values. Note that larger cluster size increases the computational complexity of our proposed algorithms. The figure demonstrates that allowing 6 RRHs serving each user is sufficient to achieve the best performance for the investigated network and parameter settings.

\section{CONCLUSION}

We have proposed a novel convex relaxation approach to solve the joint coordinated beamforming and admission control problem in fronthaul constrained cloud-RANs. Numerical results have confirmed that the proposed algorithm significantly outperforms the greedy algorithm. In addition, we have studied the impacts of various system parameters on the network performance.

\section{APPENDIX A Proof OF Proposition 1}

Proof of the first statement: The solution of problem (12) is always feasible because the feasible set is not empty. In particular, all constraints in problem (12) are satisfied if we choose $s_{u}=1$ for all $u \in \mathcal{U}$ and all transmission powers are zero. 
Proof of the second statement: We assume that there exists $\left(\mathbf{V}^{\prime},\left\{\mathbf{p}^{k \prime}\right\},\left\{s_{u}^{\prime}\right\}\right)$ which satisfies all constraints in problem (12) and $\left|\mathcal{S}^{\prime}\right|<\left|\mathcal{S}^{*}\right|$. Because $\left(\mathbf{V}^{*},\left\{\mathbf{p}^{k *}\right\},\left\{s_{u}^{*}\right\}\right)$ is the optimal solution of problem (12), we have

$$
\begin{gathered}
\sum_{k \in \mathcal{K}}\left\|\mathbf{p}^{k *}\right\|_{\mathbf{1}}+A\left|\mathcal{S}^{*}\right| \leq \sum_{k \in \mathcal{K}}\left\|\mathbf{p}^{k \prime}\right\|_{\mathbf{1}}+A\left|\mathcal{S}^{\prime}\right| \\
\rightarrow A \leq A\left(\left|\mathcal{S}^{*}\right|-\left|\mathcal{S}^{\prime}\right|\right) \leq \sum_{k \in \mathcal{K}}\left\|\mathbf{p}^{k \prime}\right\|_{\mathbf{1}}-\left\|\mathbf{p}^{k *}\right\|_{\mathbf{1}}
\end{gathered}
$$

This results in a contradiction if $A>\sum_{k \in \mathcal{K}} P_{k}$ since $\sum_{k \in \mathcal{K}}\left\|\mathbf{p}^{k \prime}\right\|_{\mathbf{1}}-\left\|\mathbf{p}^{k *}\right\|_{\mathbf{1}}$ must be less than $\sum_{k \in \mathcal{K}} P_{k}$. Hence, by choosing $A>\sum_{k \in \mathcal{K}} P_{k}$, we can minimize the number of removed users.

\section{APPENDIX B ProOF OF Proposition 3}

Proof of the first statement: Let $\Omega_{l}$ be the optimum value of problem (24)-(25) in iteration $l$. We will prove that $\Omega_{l}$ decreases over each iteration; hence, our proposed algorithm converges. Denote $\mathcal{F}_{l}$ as the feasible set of (24)-(25) in iteration $l$, which corresponds to the value of $\left\{z_{u}^{k,(l)}\right\}$. Let us define $\left(\mathbf{V}^{(l)},\left\{p_{u}^{k,(l)}\right\},\left\{s_{u}^{(l)}\right\}\right)$ as the optimal solution of problem (24)-(25) with the value of $\left\{z_{u}^{k(l)}\right\}$ as we run Algorithm 1. By setting $\left\{z_{u}^{k,(l+1)}\right\}$ as in (22), we have $\left(\mathbf{V}^{(l)},\left\{p_{u}^{k,(l)}\right\},\left\{s_{u}^{(l)}\right\}\right) \in \mathcal{F}_{l+1}$. Hence, we must have

$$
\Omega_{l} \geq \Omega_{l+1}, \quad \forall l>0,
$$

which completes the proof.

Proof of the second statement: Denote $\mathcal{F}$ as the feasible set of problems (17)-(18). Because of (20), if $\sum_{u \in \mathcal{U}} z_{u}^{k} \mathbf{v}_{u}^{k H} \mathbf{v}_{u}^{k} \leq$ $\bar{C}_{k}-\sum_{u \in \mathcal{U}}\left[R_{p}^{* u}\left(z_{u}^{k}\right)+\alpha z_{u}^{k}\right]$, we have $\sum_{u \in \mathcal{U}} R_{u}\left(p_{u}^{k}\right) \leq \bar{C}_{k}$ for any value of $\left\{z_{u}^{k(l+1)}\right\}$. Therefore, we have $\mathcal{F}_{l} \subseteq \mathcal{F}$ for iteration $l$, which means the optimal solution of problem (24)(25) in any iteration $l$ satisfies all constraints of problem (17)(18). Hence, Algorithm 1 returns the solution that satisfies all constraints of problem (17)-(18).

\section{APPENDIX C}

\section{Semi-Definite Program Form of Problem (24)-(25)}

For the particular sets $\left\{\mathcal{K}_{u}\right\}$, let $\mathbf{v}_{u}$ denote the precoding solution over RRHs in set $\mathcal{K}_{u}$ serving user $u$, which is defined as $\mathbf{v}_{u}=\left[\mathbf{v}_{u}^{u_{1} T}, \mathbf{v}_{u}^{u_{2} T}, \ldots, \mathbf{v}_{u}^{u_{a_{u}} T}\right]^{T}$ where $\left\{u_{1}, \ldots, u_{a_{u}}\right\}=\mathcal{K}_{u}$ and $a_{u}=\left|\mathcal{K}_{u}\right|$. Here, we have $\mathbf{v}_{u} \in \mathbb{C}^{N_{u} \times 1}$ where $N_{u}=$ $\sum_{k \in \mathcal{K}_{u}} N_{k}$. We are interested in determining $\mathbf{v}_{u}$ because we have $\mathbf{v}_{u}^{\imath}=\mathbf{0}$ for $i \notin \mathcal{K}_{u}$.

Let us define $\mathbf{W}_{u}=\mathbf{v}_{u} \mathbf{v}_{u}^{H}$, we have $\mathbf{W}_{u} \in \mathbb{C}^{N_{u} \times N_{u}}$. It is positive semi-definite $\left(\mathbf{W}_{u} \succeq \mathbf{0}\right)$ and has rank one because it is generated from vector $\mathbf{v}_{u}$. We also define the channel vector $\mathbf{h}_{u}^{i}=\left[\mathbf{h}_{u}^{i_{1} T}, \mathbf{h}_{u}^{i_{2} T}, \ldots, \mathbf{h}_{u}^{i_{a_{i} T} T}\right]^{T}$ and $\mathbf{H}_{u}^{i}=\mathbf{h}_{u}^{i} \mathbf{h}_{u}^{i H}$ for all $u, i \in \mathcal{U}$. Additionally, we introduce rank-one positive matrix variables $\mathbf{S}_{u}:=\mathbf{s}_{u} \mathbf{s}_{u}^{T}$ where $\mathbf{s}_{u}$ is defined as $\mathbf{s}_{u}=\left[\begin{array}{ll}s_{u} & 1\end{array}\right]^{T}$.
Then, the SINR constraint for UE $u$ in (7), the power constraints for RRHs, and the corresponding constraint with $\left\{\hat{z}_{u}^{k}\right\}$ can be rewritten in the matrix forms, respectively as

$$
\begin{array}{r}
\frac{\operatorname{Tr}\left(\mathbf{H}_{u}^{u} \mathbf{W}_{u}\right)+\mu \operatorname{Tr}\left(\mathbf{1}_{2 \times 2} \mathbf{S}_{u}\right)}{\sum_{i \in \mathcal{U} / u} \operatorname{Tr}\left(\mathbf{H}_{u}^{i} \mathbf{W}_{i}\right)+\sigma^{2}} \geq \bar{\gamma}_{u}, \quad \forall u \in \mathcal{U}, \\
\sum_{u \in \mathcal{U}} \operatorname{Tr}\left(\mathbf{E}_{u}^{k} \mathbf{W}_{u}\right) \leq P_{k}, \quad \forall k \in \mathcal{K} \\
\sum_{u \in \mathcal{U}} \operatorname{Tr}\left(\mathbf{Z}_{u}^{k} \mathbf{W}_{u}\right) \leq \bar{C}_{k}+\sum_{u \in \mathcal{U}}\left[R_{p}^{* u}\left(\hat{z}_{u}^{k}\right)-\alpha \hat{z}_{u}^{k}\right], \quad \forall k \in \mathcal{K} .
\end{array}
$$

where $\mathbf{E}_{u}^{k}=\operatorname{diag}\left(\mathbf{0}_{N_{u_{1}} \times 1}, \ldots, \mathbf{1}_{N_{u_{i}} \times 1}, \ldots, \mathbf{0}_{N_{u_{a_{u}}} \times 1}\right)$ and $\mathbf{Z}_{u}^{k}=\operatorname{diag}\left(\mathbf{0}_{N_{u_{1}} \times 1}, \ldots, \hat{z}_{u}^{u_{i}} \mathbf{1}_{N_{u_{i}} \times 1}, \ldots, \mathbf{0}_{N_{u_{a_{u}}} \times 1}\right)$ if $u_{i}=k$. Therefore, the weighted sum-power minimization can be formulated as the following SDP problem

$$
\begin{aligned}
\min _{\left\{\mathbf{W}_{u}\right\}_{u=1}^{M}} & \sum_{u=1}^{M} \operatorname{Tr}\left(\mathbf{D}_{u} \mathbf{W}_{u}\right)+(A / 4) \sum_{u=1}^{M} \operatorname{Tr}\left(\mathbf{1}_{2 \times 2} \mathbf{S}_{u}\right) \\
\text { s.t. } & \text { constraints (28), (29), and (30) } \\
& \mathbf{W}_{u} \succeq \mathbf{0}, \operatorname{rank}\left(\mathbf{W}_{u}\right)=1, \forall u \\
& \mathbf{S}_{u} \succeq \mathbf{0}, \operatorname{rank}\left(\mathbf{S}_{u}\right)=1, \mathbf{S}_{u}(1,1)=\mathbf{S}_{u}(2,2)=1, \forall u
\end{aligned}
$$

This transformation reveals structure of the underlying problem. Specifically, if we remove the rank-one constraints $\operatorname{rank}\left(\mathbf{W}_{u}\right)=1$ and $\operatorname{rank}\left(\mathbf{S}_{u}\right)=1$ from (31) then the resulting problem is convex. As given in Theorem 3.1 of [12], if (31) is feasible, then it has at least one solution where $\operatorname{rank}\left(\mathbf{W}_{u}\right)=1$, for all $u \in \mathcal{U}$. Then, $\mathbf{v}_{u}$ can be calculated as the eigenvector of $\mathbf{W}_{u}$, and $s_{u}$ can be calculated from $\mathbf{S}_{u}$.

\section{REFERENCES}

[1] M. Sawahashi, Y. Kishiyama, A. Morimoto, D. Nishikawa, and M. Tanno, "Coordinated multipoint transmission/reception techniques for LTE-advanced [Coordinated and distributed MIMO]," IEEE Wireless Commun., vol. 17, no. 3, pp. 26-34, Jun. 2010.

[2] P. Marsch and G. Fettweis, "Uplink CoMP under a constrained backhaul and imperfect channel knowledge," IEEE Trans. Wireless Commun., vol. 10, no. 6, pp. 1730-1742, Jun. 2011.

[3] "C-RAN: The road towards green ran," White Paper, China Mobile, 2011.

[4] E. Hossain, L. B. Le, and D. Niyato. Radio Resource Management in Multi-Tier Cellular Wireless Networks. Wiley, 2013.

[5] $\mathrm{Vu} \mathrm{N}$. Ha and Long B. Le, "Distributed base station association and power control for heterogeneous cellular networks," IEEE Trans. Veh. Technol., vol. 63, no. 1, pp. 282-296, Jan. 2014.

[6] S.-H. Park, O. Simeone, O. Sahin and S. Shamai (Shitz), "Robust layered transmission and compression for distributed uplink reception in cloud radio access networks," IEEE Trans. Veh. Technol., vol. 63, no. 1, pp. 204-216, Jan. 2014.

[7] R. Zakhour and D. Gesbert, "Optimized data sharing in multicell MIMO with finite backhaul capacity," IEEE Trans. Signal Process., vol. 59, no. 12, pp. 6102-6111, Dec. 2011.

[8] Y. Shi, J. Zhang, and K. B. Letaief, "Group sparse beamforming for green cloud radio access networks," in Proc. GLOBECOM, 2013.

[9] Vu N. Ha, L. B. Le, and N.-D. Dao, "Cooperative transmission in Cloud RAN considering fronthaul capacity and cloud processing constraints," in Proc. IEEE WCNC'2014, Apr. 2014.

[10] Vu N. Ha, L. B. Le, and N.-D. Dao, "Energy-efficient coordinated transmission for Cloud-RANs: Algorithm design and tradeoff," in Proc. IEEE CISS'2014, Mar. 2014.

[11] E. Matskani, N. Sidiropoulos, Z.-Q. Luo, and L. Tassiulas, "Convex approximation techniques for joint multiuser downlink beamforming and admission control," IEEE Trans. Wireless Commun., vol. 7, pp. 26822693, July 2008.

[12] M. Bengtsson and B. Ottersten, "Optimal downlink beamforming using semidefinite optimization," in Proc. Annual Allerton Conf. on Commun., Control and Computing, pp. 987-996, Sept. 1999. 